\title{
QUARCH: A New Quasi-Affine Reconstruction Stratum From Vague Relative Camera Orientation Knowledge
}

\author{
Devesh Adlakha ${ }^{1,3}$, Adlane Habed ${ }^{1}$, Fabio Morbidi ${ }^{2}$, Cédric Demonceaux ${ }^{3}$, Michel de Mathelin ${ }^{1}$ \\ ${ }^{1}$ ICube laboratory, CNRS, University of Strasbourg \\ ${ }^{2}$ MIS laboratory, University of Picardie Jules Verne \\ ${ }^{3}$ ImViA laboratory, VIBOT ERL CNRS, University of Burgundy - Franche-Comté \\ adlakha@unistra.fr
}

\begin{abstract}
We present a new quasi-affine reconstruction of a scene and its application to camera self-calibration. We refer to this reconstruction as QUARCH (QUasi-Affine Reconstruction with respect to Camera centers and the Hodographs of horopters). A QUARCH can be obtained by solving a semidefinite programming problem when, $(i)$ the images have been captured by a moving camera with constant intrinsic parameters, and (ii) a vague knowledge of the relative orientation (under or over $120^{\circ}$ ) between camera pairs is available. The resulting reconstruction comes close enough to an affine one allowing thus an easy upgrade of the QUARCH to its affine and metric counterparts. We also present a constrained Levenberg-Marquardt method for nonlinear optimization subject to Linear Matrix Inequality (LMI) constraints so as to ensure that the QUARCH LMIs are satisfied during optimization. Experiments with synthetic and real data show the benefits of $Q U A R C H$ in reliably obtaining a metric reconstruction.
\end{abstract}

\section{Introduction}

In multi-view computer vision, accurately locating the plane at infinity $\left(\Pi_{\infty}\right)$ is considered crucial for successfully lifting a projective structure and cameras to a metric frame [20, 5]. Locating $\Pi_{\infty}$ reliably has proved challenging in camera self-calibration due to the nonlinearity of the problem [11, 12]. Once it is located, the calibration parameters may be obtained by solving linear equations for the (dual) image of the absolute conic. When camera parameters are constant, a necessary condition on $\Pi_{\infty}$ is that the eigenvalues of its inter-image homography matrices have equal moduli. This so-called modulus constraint [20] leads to quartic polynomial equations in the coordinates of $\Pi_{\infty}$

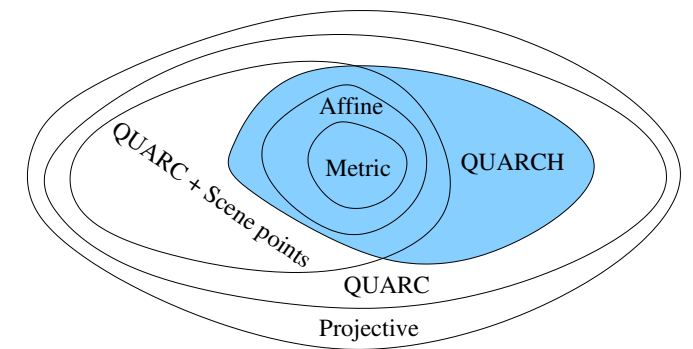

Figure 1: QUARCH is a specialization of the QUARC stratum and is therefore one step closer to the affine stratum.

for which several solutions have been proposed [20, 8, 5].

Other methods [11, 9] locate $\Pi_{\infty}$ by first upgrading a projective reconstruction to a quasi-affine one based on Hartley's cheirality theory [10]. In [11], the cheirality inequalities are used to obtain bounds on the coordinates of $\Pi_{\infty}$, which is then located through an exhaustive search within these bounds. The quasi-affine reconstruction in [11, 9] is with respect to the set of camera centers and that of scene points: the sets whose respective convex hulls are preserved. Nistér [17] pointed out that scene points may not be reliable and therefore sought a quasi-affine reconstruction with respect to camera centers (QUARC) alone. A QUARC is upgraded to a metric reconstruction through nonlinear optimization of a geometrically meaningful cost function derived from priors on the calibration parameters.

In this paper, we show the existence of a new quasiaffine reconstruction stratum that we refer to as QUARCH: QUasi-Affine Reconstruction with respect to Camera centers and the Hodographs of horopters. A QUARCH is a specialization of a QUARC (see Figure 1) that additionally satisfies a new set of relative camera orientation-based convex constraints on $\Pi_{\infty}$. These constraints are formulated as Linear Matrix Inequalities (LMIs) and they describe 
the relationship of $\Pi_{\infty}$ with the hodographs [21, 2] of the horopters [24, 23] of camera pairs. A QUARCH can be obtained when the calibration parameters are constant and when the relative orientation angle between a set of camera pairs is known to be either under or over $120^{\circ}$.

We use QUARCH in a self-calibration algorithm as a first step towards obtaining a metric reconstruction from a projective one. To obtain a QUARCH, our assumption is that the relative orientation angle between consecutive views is under $120^{\circ}$. This is a mild assumption that is often implicitly verified when capturing images so as to ensure a sufficient overlap for feature matching. $\Pi_{\infty}$ is then located through nonlinear optimization of a suitable cost function, such as using the modulus constraints. We also propose a constrained Levenberg-Marquardt (LM) method for nonlinear optimization subject to LMI constraints. This ensures that the QUARCH LMIs are satisfied during the local optimization. Our experiments show that a QUARCH plane is an excellent starting plane for our algorithm to reliably converge to $\Pi_{\infty}$ and that constraining the local optimization to satisfy the QUARCH LMIs further improves the success rate of locating it. The main contributions of this paper are:

- QUARCH: a new quasi-affine stratum along with an algorithm for camera self-calibration based on it.

- Constrained LM method: an LM-type algorithm for nonlinear optimization subject to LMI constraints.

Notation: We consider the scene embedded in the projective 3 -space $\mathbb{P}^{3}$. A point $X$ and plane $\Pi$ in $\mathbb{P}^{3}$ are represented by 4 -dimensional homogeneous column vectors, $\mathrm{X}$ and $\Pi$, respectively. The plane at infinity is referred to as $\Pi_{\infty}$ and its coordinates by $\Pi_{\infty}$. A perspective camera is represented by its $3 \times 4$ projection matrix P. Finally, $(\cdot)_{k}$ refers to the $k$-th entry of its vector argument, $\mathrm{I}_{n}$ is the $n \times n$ identity matrix, $0_{d}$ the $d$-dimensional zero vector, $\operatorname{sgn}(\cdot)$ the sign function, and $\simeq$ the equality up to scale.

\section{Background}

This section is a brief review of key results from the literature and of LMIs, which our work is based upon.

\subsection{Modulus constraint and the horopter}

Shaffalitzky [24] showed the connection between the modulus constraint and the horopter curves. The horopter $\mathcal{H}$ of a camera pair with identical calibration parameters is the locus of points in $\mathbb{P}^{3}$ that are imaged at the same coordinates by both cameras. As such, a point $X$ on the horopter satisfies $\mathrm{P}_{i} \mathrm{X} \simeq \mathrm{P}_{j} \mathrm{X}$, and the locus of these points is $\mathcal{H}(s, t) \triangleq \mathcal{N}\left(s \mathrm{P}_{i}-t \mathrm{P}_{j}\right)$ for parameters $s$ and $t$, where $\mathcal{N}(\cdot)$ is the algebraic nullspace operator defined in [24] as $\operatorname{det}\left(\left[\begin{array}{ll}\mathrm{P}^{\top} & \Pi\end{array}\right]\right)=\Pi^{\top} \mathcal{N}(\mathrm{P})$ for any plane $\Pi$. The algebraic nullspace defines the scale of the null vector $\mathcal{N}(\mathrm{P})$ from the scale of the matrix P. The parametric form of the horopter of the camera pair $(i, j)$ is:

$$
\mathcal{H}(s, t)=s^{3} \mathrm{C}_{i}-s^{2} t \mathrm{~T}_{i j}+s t^{2} \mathrm{~T}_{j i}-t^{3} \mathrm{C}_{j},
$$

where $\mathrm{C}_{i}=\mathcal{N}\left(\mathrm{P}_{i}\right)$ and $\mathrm{C}_{j}=\mathcal{N}\left(\mathrm{P}_{j}\right)$ are the two camera centers, $\mathrm{T}_{i j}=\mathcal{T}\left(\mathrm{P}_{i}, \mathrm{P}_{j}\right), \mathrm{T}_{j i}=\mathcal{T}\left(\mathrm{P}_{j}, \mathrm{P}_{i}\right)$, and operator $\mathcal{T}$ is defined by this expansion. The horopter is a twisted cubic in $\mathbb{P}^{3}$ that passes through both camera centers and intersects a plane (including $\Pi_{\infty}$ ) at three points. From [24], we have:

$$
\Pi_{\infty}^{\mathrm{T}}\left[\mathrm{C}_{i} \mathrm{~T}_{i j} \mathrm{~T}_{j i} \mathrm{C}_{j}\right]=\left(\lambda_{i}^{3}, \lambda_{i}^{2} \lambda_{j} a_{i j}, \lambda_{i} \lambda_{j}^{2} a_{i j}, \lambda_{j}^{3}\right),
$$

where $\lambda_{i}$ and $\lambda_{j}$ are the scale factors of $\mathrm{P}_{i}$ and $\mathrm{P}_{j}$, respectively, and $a_{i j}=1+2 \cos \theta_{i j}$, with $\theta_{i j}$ being the relative orientation angle between the two cameras. Eliminating the scalars from (2) leads to the modulus constraint:

$$
\mathcal{M}_{i j} \triangleq \Pi_{\infty}^{\top} \mathrm{C}_{i}\left(\Pi_{\infty}^{\mathrm{T}} \mathrm{T}_{j i}\right)^{3}-\Pi_{\infty}^{\mathrm{T}} \mathrm{C}_{j}\left(\Pi_{\infty}^{\mathrm{T}} \mathrm{T}_{i j}\right)^{3}=0,
$$

that is a quartic polynomial equation first derived in [20].

\subsection{Sign-corrected cameras and QUARC}

Scene points that appear in front of two cameras in the true metric reconstruction may appear in front of one camera but behind the other in a projective reconstruction. Such a camera pair is said to be twisted by a projective transformation and untwisted otherwise. In a twisted camera pair, $\Pi_{\infty}$ intersects the baseline of the two cameras. A QUARC (QUasi-Affine Reconstruction with respect to Camera centers) [17] is a projective reconstruction that does not contain any twisted pairs. A projective reconstruction may be upgraded to a QUARC using the following two steps: (i) correct the signs $\zeta_{i} \in\{-1,1\}$ of the projection matrices, $\tilde{\mathrm{P}}_{i}=\zeta_{i} \mathrm{P}_{i}$, such that all camera centers lie on one side with respect to $\Pi_{\infty}$, so that $\Pi_{\infty}^{\mathrm{T}} \tilde{\mathrm{C}}_{i}>0$ [17, Alg. 2], and (ii) map to infinity a plane that carries the same signature as $\Pi_{\infty}$ with respect to the camera centers, that is a plane that satisfies $\Pi^{\mathrm{T}} \tilde{\mathrm{C}}_{i}>0$. Such a QUARC plane can be found by solving a Linear Programming (LP) problem [17, Sec. 8].

\subsection{Linear Matrix Inequalities}

A Linear Matrix Inequality (LMI) is a constraint on a vector $x=\left(x_{1}, \ldots, x_{m}\right)^{\top} \in \mathbb{R}^{m}$ such that $F(x) \succeq 0$, where $F(x) \triangleq F_{0}+\sum_{i=1}^{m} F_{i} x_{i}$ is an affine function of $x$ involving symmetric matrices $F_{0}, \ldots, F_{m} \in \mathbb{R}^{n \times n}$. The LMI $F(x) \succeq 0$ means that $F(x)$ is positive semidefinite. The LMI may also be strict, in which case $F(x)$ is positive definite. Convex quadratic inequalities may be reformulated into LMIs by using the Schur complement lemma [3]:

Lemma 2.1. Given a real symmetric block-partitioned matrix $D=\left[\begin{array}{cc}A & B \\ B^{\top} & C\end{array}\right]$ and the Schur complement $S=C-$ $B^{\top} A^{-1} B$ of (the symmetric block) $A$ in $D$,

(i) if $A \succ 0$, then $D \succeq 0$ if and only if $S \succeq 0$.

(ii) $D \succ 0$ if and only if $A \succ 0$ and $S \succ 0$. 


\section{A new quasi-affine reconstruction stratum}

In this section, we introduce the theory behind QUARCH and present an algorithm for camera self-calibration based on it. In Section 3.1. we characterize the relationship of $\Pi_{\infty}$ with the hodographs of the horopter based on the relative orientation between a camera pair. From this characterization, we derive a new set of LMI constraints on $\Pi_{\infty}$ in Section 3.2. In Section 3.3, these constraints are used to obtain a QUARCH. Finally, in Section 3.4, we detail our algorithm to upgrade QUARCH to a metric reconstruction.

\subsection{Hodographs of the horopter}

Definition 3.1 (Hodographs of the horopter). For a camera pair $(i, j)$ with attached horopter $\mathcal{H}$, the hodographs $\mathcal{H}_{s}$ and $\mathcal{H}_{t}$ of $\mathcal{H}$ are the curves traced out by the partial derivatives of $\mathcal{H}$ in $\mathbb{P}^{3}$. Let $\mathcal{H}_{s}(s, t) \triangleq \frac{\partial \mathcal{H}(s, t)}{\partial s}$ and $\mathcal{H}_{t}(s, t) \triangleq \frac{\partial \mathcal{H}(s, t)}{\partial t}$, the parametric forms of these curves are:

$$
\begin{aligned}
& \mathcal{H}_{s}(s, t)=3 s^{2} \mathrm{C}_{i}-2 s t \mathrm{~T}_{i j}+t^{2} \mathrm{~T}_{j i}, \\
& \mathcal{H}_{t}(s, t)=-3 t^{2} \mathrm{C}_{j}+2 s t \mathrm{~T}_{j i}-s^{2} \mathrm{~T}_{i j} .
\end{aligned}
$$

Observe that $\mathcal{H}_{s}(s, t)$ passes through the points $\mathrm{C}_{i}$ and $\mathrm{T}_{j i}$, while $\mathcal{H}_{t}(s, t)$ passes through $\mathrm{C}_{j}$ and $\mathrm{T}_{i j}$. Hereafter, we simply refer to the hodographs of the horopter as the hodographs. We are now interested in characterizing the relationship of $\Pi_{\infty}$ with the hodographs. To do so, we consider sign-corrected projection matrices $\tilde{\mathrm{P}}_{i}$ and $\tilde{\mathrm{P}}_{j}$. Their associated horopter $\mathcal{H}$ is represented by $\tilde{\mathcal{H}}(s, t)=s^{3} \tilde{\mathrm{C}}_{i}-$ $s^{2} t \tilde{\mathrm{T}}_{i j}+s t^{2} \tilde{\mathrm{T}}_{j i}-t^{3} \tilde{\mathrm{C}}_{j}$, obtained from the expansion of $\tilde{\mathcal{H}}(s, t) \triangleq \mathcal{N}\left(s \tilde{\mathrm{P}}_{i}-t \tilde{\mathrm{P}}_{j}\right)$. The corresponding hodographs $\mathcal{H}_{s}$ and $\mathcal{H}_{t}$ are represented by $\tilde{\mathcal{H}}_{s}(s, t)$ and $\tilde{\mathcal{H}}_{t}(s, t)$. Given this representation, we first characterize the relationship of $\Pi_{\infty}$ with the points $\left(\tilde{\mathrm{T}}_{i j}, \tilde{\mathrm{T}}_{j i}\right)$ through the following lemma.

Lemma 3.1. For a camera pair $(i, j)$ with relative orientation angle $\theta_{i j}$ and attached horopter $\mathcal{H}$, the plane at infinity $\Pi_{\infty}$ satisfies the following linear inequalities:

$$
\begin{aligned}
& \Pi_{\infty}^{\top} \tilde{\mathrm{T}}_{i j} \geq 0 \text { and } \Pi_{\infty}^{\mathrm{T}} \tilde{\mathrm{T}}_{j i} \geq 0 \text { if }\left|\theta_{i j}\right| \leq 120^{\circ}, \\
& \Pi_{\infty}^{\top} \tilde{\mathrm{T}}_{i j} \leq 0 \text { and } \Pi_{\infty}^{\mathrm{T}} \tilde{\mathrm{T}}_{j i} \leq 0 \text { if }\left|\theta_{i j}\right| \geq 120^{\circ},
\end{aligned}
$$

where the equality holds for $\left|\theta_{i j}\right|=120^{\circ}$.

Proof. From (2), observe that $\Pi_{\infty}^{\top} \tilde{\mathrm{T}}_{i j}$ and $\Pi_{\infty}^{\top} \tilde{\mathrm{T}}_{j i}$ depend on $\theta_{i j}$. For sign-corrected projection matrices, $\Pi_{\infty}^{\top} \tilde{\mathrm{C}}_{i}>0$ and $\Pi_{\infty}^{\top} \tilde{\mathrm{C}}_{j}>0$, hence $\lambda_{i}$ and $\lambda_{j}$ are positive. Thus, $\operatorname{sgn}\left(\Pi_{\infty}^{\mathbf{T}} \tilde{T}_{i j}\right)=\operatorname{sgn}\left(\Pi_{\infty}^{\mathrm{T}} \tilde{\mathrm{T}}_{j i}\right)=\operatorname{sgn}\left(a_{i j}\right)$, where $a_{i j}$ :

$$
\begin{array}{r}
0 \leq a_{i j} \leq 3 \text { if }\left|\theta_{i j}\right| \leq 120^{\circ}, \\
-1 \leq a_{i j} \leq 0 \text { if }\left|\theta_{i j}\right| \geq 120^{\circ},
\end{array}
$$

and $a_{i j}=0$ if $\left|\theta_{i j}\right|=120^{\circ}$.
The linear inequalities in (5) form a new set of relative camera orientation-based constraints on $\Pi_{\infty}$. They signify that the (virtual) points $\tilde{\mathrm{T}}_{i j}$ and $\tilde{\mathrm{T}}_{j i}$ lie on the same side as the camera centers with respect to $\Pi_{\infty}$ when $\left|\theta_{i j}\right|<120^{\circ}$, whereas they lie on the opposite side when $\left|\theta_{i j}\right|>120^{\circ}$. Imposing these inequalities for a set of camera pairs in the QUARC LP problem leads to a QUARC that is additionally quasi-affine with respect to the corresponding set of points $\left(\tilde{\mathrm{T}}_{i j}, \tilde{\mathrm{T}}_{j i}\right)$. We now extend this incidence relationship of $\Pi_{\infty}$ with $\left(\widetilde{\mathrm{T}}_{i j}, \tilde{\mathrm{T}}_{j i}\right)$ to the hodographs that contain them.

Lemma 3.2. For a camera pair $(i, j)$ with relative orientation angle $\theta_{i j}$ and attached horopter $\mathcal{H}$, the plane at infinity $\Pi_{\infty}$ intersects the hodographs $\mathcal{H}_{s}$ and $\mathcal{H}_{t}$ in: (i) at most one real point if $\left|\theta_{i j}\right| \leq 120^{\circ}$, and (ii) at least one real point if $\left|\theta_{i j}\right| \geq 120^{\circ}$.

Proof. Consider the equations representing the intersection of $\Pi_{\infty}$ with the hodographs:

$$
\begin{aligned}
& \Pi_{\infty}^{\top} \tilde{\mathcal{H}}_{s}(s, t)=3 s^{2} \Pi_{\infty}^{\top} \tilde{\mathrm{C}}_{i}-2 s t \Pi_{\infty}^{\top} \tilde{\mathrm{T}}_{i j}+t^{2} \Pi_{\infty}^{\top} \tilde{\mathrm{T}}_{j i}, \\
& \Pi_{\infty}^{\top} \tilde{\mathcal{H}}_{t}(s, t)=-3 t^{2} \Pi_{\infty}^{\top} \tilde{\mathrm{C}}_{j}+2 s t \Pi_{\infty}^{\top} \tilde{\mathrm{T}}_{j i}-s^{2} \Pi_{\infty}^{\top} \tilde{\mathrm{T}}_{i j} .
\end{aligned}
$$

These equations are quadratic in $s$ and $t$, respectively. Therefore, their discriminant functions $\Delta_{s}$ and $\Delta_{t}$,

$$
\begin{aligned}
\Delta_{s} & =-4 t^{2}\left(3\left(\Pi_{\infty}^{\top} \tilde{\mathrm{T}}_{j i}\right)\left(\Pi_{\infty}^{\top} \tilde{\mathrm{C}}_{i}\right)-\left(\Pi_{\infty}^{\top} \tilde{\mathrm{T}}_{i j}\right)^{2}\right) \\
\Delta_{t} & =-4 s^{2}\left(3\left(\Pi_{\infty}^{\top} \tilde{\mathrm{T}}_{i j}\right)\left(\Pi_{\infty}^{\top} \tilde{\mathrm{C}}_{j}\right)-\left(\Pi_{\infty}^{\top} \tilde{\mathrm{T}}_{j i}\right)^{2}\right)
\end{aligned}
$$

characterize the intersection. Recall that the discriminant is negative for no real points of intersection, positive for two real points of intersection, and zero for one real point of intersection. Substituting the values from (2), we have that:

$$
\begin{aligned}
& \Delta_{s}=-4 t^{2} \lambda_{i}^{4} \lambda_{j}^{2} a_{i j}\left(3-a_{i j}\right), \\
& \Delta_{t}=-4 s^{2} \lambda_{i}^{2} \lambda_{j}^{4} a_{i j}\left(3-a_{i j}\right) .
\end{aligned}
$$

The discriminant functions $\Delta_{s}$ and $\Delta_{t}$ depend on $a_{i j}$ and thus on $\theta_{i j}$. From (6), we can deduce that:

$$
\begin{aligned}
& \Delta_{s} \leq 0 \text { and } \Delta_{t} \leq 0 \text { if }\left|\theta_{i j}\right| \leq 120^{\circ}, \\
& \Delta_{s} \geq 0 \text { and } \Delta_{t} \geq 0 \text { if }\left|\theta_{i j}\right| \geq 120^{\circ},
\end{aligned}
$$

where $\Delta_{s}=0$ and $\Delta_{t}=0$ for $\left|\theta_{i j}\right| \in\left\{0^{\circ}, 120^{\circ}\right\}$, since $a_{i j}=0$ if $\left|\theta_{i j}\right|=120^{\circ}$, and $a_{i j}=3$ if $\theta_{i j}=0^{\circ}$.

The incidence relationship of $\Pi_{\infty}$ with the hodographs of a camera pair is thus determined by the relative orientation angle between the two cameras. Their intersection is in at most one real point if $\left|\theta_{i j}\right| \leq 120^{\circ}$, whereas it is in at least one real point if $\left|\theta_{i j}\right| \geq 120^{\circ}$. The hodographs thus act as "virtual positioning objects" for $\Pi_{\infty}$. 


\subsection{LMI constraints on the plane at infinity}

We now derive necessary conditions on $\Pi_{\infty}$ based on its relationship with the hodographs given in Lemma 3.2

Proposition 3.3 (Case of $\left|\theta_{i j}\right| \leq 120^{\circ}$ ). For a camera pair $(i, j)$ with relative orientation angle $\left|\theta_{i j}\right| \leq 120^{\circ}$ and horopter $\mathcal{H}$, the plane at infinity $\Pi_{\infty}$ satisfies the two LMIs:

$$
\left[\begin{array}{cc}
\Pi_{\infty}^{\mathrm{T}} \tilde{\mathrm{C}}_{i} & \Pi_{\infty}^{\mathrm{T}} \tilde{\mathrm{T}}_{i j} \\
\Pi_{\infty}^{\mathrm{T}} \tilde{\mathrm{T}}_{i j} & 3 \Pi_{\infty}^{\mathrm{T}} \tilde{\mathrm{T}}_{j i}
\end{array}\right] \succeq 0,\left[\begin{array}{cc}
\Pi_{\infty}^{\mathrm{T}} \tilde{\mathrm{C}}_{j} & \Pi_{\infty}^{\mathrm{T}} \tilde{\mathrm{T}}_{j i} \\
\Pi_{\infty}^{\top} \tilde{\mathrm{T}}_{j i} & 3 \Pi_{\infty}^{\mathrm{T}} \tilde{\mathrm{T}}_{i j}
\end{array}\right] \succeq 0 .
$$

Proof. From Lemma 3.2, when $\left|\theta_{i j}\right| \leq 120^{\circ}, \Pi_{\infty}$ intersects each hodograph in at most one real point. This implies that the discriminants $\Delta_{s}$ and $\Delta_{t}$ ought to be nonpositive. The proof herein boils down to showing that these discriminants are so when 111 is true. To show this, consider the Schur complements $\mathcal{S}_{i j}\left(\Pi_{\infty}\right)$ and $\mathcal{S}_{j i}\left(\Pi_{\infty}\right)$ of $\Pi_{\infty}^{\top} \tilde{\mathrm{C}}_{i}$ and $\Pi_{\infty}^{\top} \tilde{\mathrm{C}}_{j}$, respectively, in the $1^{\text {st }}$ and $2^{\text {nd }}$ matrix of (11):

$$
\begin{aligned}
& \mathcal{S}_{i j}\left(\Pi_{\infty}\right) \triangleq 3 \Pi_{\infty}^{\mathrm{T}} \tilde{\mathrm{T}}_{j i}-\left(\Pi_{\infty}^{\mathrm{T}} \tilde{\mathrm{T}}_{i j}\right)^{2}\left(\Pi_{\infty}^{\mathrm{T}} \tilde{\mathrm{C}}_{i}\right)^{-1}, \\
& \mathcal{S}_{j i}\left(\Pi_{\infty}\right) \triangleq 3 \Pi_{\infty}^{\mathrm{T}} \tilde{\mathrm{T}}_{i j}-\left(\Pi_{\infty}^{\mathrm{T}} \tilde{\mathrm{T}}_{j i}\right)^{2}\left(\Pi_{\infty}^{\mathrm{T}} \tilde{\mathrm{C}}_{j}\right)^{-1} .
\end{aligned}
$$

Rewriting the discriminant functions in $(8)$ in terms of these Schur complements, we have that:

$$
\begin{aligned}
& \Delta_{s}=-4 t^{2} \Pi_{\infty}^{\top} \tilde{\mathrm{C}}_{i} \mathcal{S}_{i j}\left(\Pi_{\infty}\right), \\
& \Delta_{t}=-4 s^{2} \Pi_{\infty}^{\top} \tilde{\mathrm{C}}_{j} \mathcal{S}_{j i}\left(\Pi_{\infty}\right) .
\end{aligned}
$$

Therefore, we can deduce that $\mathcal{S}_{i j}\left(\Pi_{\infty}\right) \geq 0$ and $\mathcal{S}_{j i}\left(\Pi_{\infty}\right) \geq 0$ for $\Delta_{s}$ and $\Delta_{t}$ to be nonpositive, since $\Pi_{\infty}^{\top} \tilde{\mathrm{C}}_{i}>0$ and $\Pi_{\infty}^{\top} \tilde{\mathrm{C}}_{j}>0$. From Lemma 2.1. (11) is true if and only if $\mathcal{S}_{i j}\left(\Pi_{\infty}\right)$ and $\mathcal{S}_{j i}\left(\Pi_{\infty}\right)$ are nonnegative.

The inequalities in 5a are necessary conditions for $a_{i j}$ to be nonnegative when $\left|\theta_{i j}\right| \leq 120^{\circ}$, whereas the LMIs in (11) are necessary conditions to further bound $a_{i j}$, as in (6a), such that $0 \leq a_{i j} \leq 3$. To see this, by partially substituting (2) in (12), we have that:

$$
\begin{aligned}
& \mathcal{S}_{i j}\left(\Pi_{\infty}\right)=\Pi_{\infty}^{\top} \tilde{\mathrm{T}}_{j i}\left(3-a_{i j}\right), \\
& \mathcal{S}_{j i}\left(\Pi_{\infty}\right)=\Pi_{\infty}^{\top} \tilde{\mathrm{T}}_{i j}\left(3-a_{i j}\right) .
\end{aligned}
$$

Now consider the $1^{\text {st }}$ LMI in (11), which imposes that $\Pi_{\infty}^{\top} \tilde{\mathrm{T}}_{j i} \geq 0$ and $\mathcal{S}_{i j}\left(\Pi_{\infty}\right) \geq 0$. It follows from 14a that $3-a_{i j} \geq 0$ and thus $0 \leq a_{i j} \leq 3$. Note that this constraint is neither enforced by the modulus constraint nor by the QUARC inequalities. With a similar argument using $14 \mathrm{~b}$, the $2^{\text {nd }}$ LMI in 11] can also be shown to be a necessary condition for $0 \leq a_{i j} \leq 3$. The following proposition completes the set of LMI constraints on $\Pi_{\infty}$.

Proposition 3.4 (Case of $\left|\theta_{i j}\right| \geq 120^{\circ}$ ). For a camera pair $(i, j)$ with relative orientation angle $\left|\theta_{i j}\right| \geq 120^{\circ}$ and horopter $\mathcal{H}$, the plane at infinity $\Pi_{\infty}$ satisfies the two LMIs:

$$
\left[\begin{array}{cc}
\Pi_{\infty}^{\mathrm{T}} \tilde{\mathrm{C}}_{i} & \Pi_{\infty}^{\mathrm{T}} \tilde{\mathrm{T}}_{i j} \\
\Pi_{\infty}^{\mathrm{T}} \tilde{\mathrm{T}}_{i j} & -\Pi_{\infty}^{\mathrm{T}} \tilde{\mathrm{T}}_{j i}
\end{array}\right] \succeq 0, \quad\left[\begin{array}{cc}
\Pi_{\infty}^{\mathrm{T}} \tilde{\mathrm{C}}_{j} & \Pi_{\infty}^{\mathrm{T}} \tilde{\mathrm{T}}_{j i} \\
\Pi_{\infty}^{\mathrm{T}} \tilde{\mathrm{T}}_{j i} & -\Pi_{\infty}^{\mathrm{T}} \tilde{\mathrm{T}}_{i j}
\end{array}\right] \succeq 0 .
$$

Proof. From Lemma 3.2, when $\left|\theta_{i j}\right| \geq 120^{\circ}, \Pi_{\infty}$ intersects each hodograph in at least one real point, therefore the discriminants $\Delta_{s}$ and $\Delta_{t}$ are nonnegative. From (13), we can deduce that $\mathcal{S}_{i j}\left(\Pi_{\infty}\right) \leq 0$ and $\mathcal{S}_{j i}\left(\Pi_{\infty}\right) \leq 0$. For the LMIs in (15) to be satisfied, the following inequalities must hold:

$$
\mathcal{S}_{i j}\left(\Pi_{\infty}\right)-4 \Pi_{\infty}^{\top} \tilde{\mathrm{T}}_{j i} \geq 0, \mathcal{S}_{j i}\left(\Pi_{\infty}\right)-4 \Pi_{\infty}^{\top} \tilde{\mathrm{T}}_{i j} \geq 0 .
$$

Note that the left-hand sides of these two inequalities are the Schur complements of $\Pi_{\infty}^{\top} \tilde{\mathrm{C}}_{i}$ and $\Pi_{\infty}^{\top} \tilde{\mathrm{C}}_{j}$, respectively, in the $1^{\text {st }}$ and $2^{\text {nd }}$ matrix of (15). Using (14), they can be rewritten as $-\Pi_{\infty}^{\top} \tilde{\mathrm{T}}_{j i}\left(1+a_{i j}\right)$ and $-\Pi_{\infty}^{\mathrm{T}} \tilde{\mathrm{T}}_{i j}\left(1+a_{i j}\right)$, respectively. From $5 \mathrm{~b}$, and $6 \mathrm{~b}$, these Schur complements are nonnegative when $\left|\theta_{i j}\right| \geq 120^{\circ}$. Hence, the inequalities in (16) hold and so do the LMIs in (15).

The linear inequalities in $5 \mathrm{~b}$ ) alone ensure that $\Delta_{s} \geq 0$ and $\Delta_{t} \geq 0$, and hence that $\Pi_{\infty}$ intersects each hodograph in at least one real point. The LMIs in 15 further characterize the region of intersection. This region is dependent on the discriminants, which, from (13), can be seen to be constrained by (16) and hence by the LMIs in (15).

The LMIs in (11) and (15) are necessary conditions on $\Pi_{\infty}$ to satisfy the incidence relationship with the hodographs given in Lemma 3.2. In the following section, we show how a vague knowledge of the relative orientation $\theta_{i j}$ between camera pairs (i.e. $\left|\theta_{i j}\right| \leq 120^{\circ}$ or $\left|\theta_{i j}\right| \geq 120^{\circ}$ ) can be exploited with these LMI constraints to obtain a new quasi-affine reconstruction of a scene: a QUARCH.

\subsection{QUARCH}

A QUARCH is a specialization of a QUARC that is additionally quasi-affine with respect to the hodographs of a set of camera pairs. A QUARCH can be obtained from a projective reconstruction following the steps outlined for QUARC in Section 2.2, but by locating a QUARCH plane instead in the second step. A QUARCH plane is a QUARC plane that additionally satisfies LMIs (11) and/or (15) for a set of camera pairs. It can be computed by solving the following Semidefinite Programming (SDP) problem:

$$
\begin{aligned}
& \max _{\Pi, \delta} \delta \\
& \text { s.t. } \quad \Pi^{\top} \tilde{\mathrm{C}}_{l} /\left\|\tilde{\mathrm{C}}_{l}\right\|>\delta, \quad l=1, \ldots, n \text {, } \\
& -1 \leq(\Pi)_{k} \leq 1, \quad k=1, \ldots, 4, \\
& {\left[\begin{array}{ll}
\Pi^{\mathrm{T}} \tilde{\mathrm{C}}_{i} & \Pi^{\mathrm{T}} \tilde{\mathrm{T}}_{i j} \\
\Pi^{\mathrm{T}} \tilde{\mathrm{T}}_{i j} & 3 \Pi^{\mathrm{T}} \tilde{\mathrm{T}}_{j i}
\end{array}\right] \succeq 0, \quad\left[\begin{array}{ll}
\Pi^{\mathrm{T}} \tilde{\mathrm{C}}_{j} & \Pi^{\mathrm{T}} \tilde{\mathrm{T}}_{j i} \\
\Pi^{\mathrm{T}} \tilde{\mathrm{T}}_{j i} & 3 \Pi^{\mathrm{T}} \tilde{\mathrm{T}}_{i j}
\end{array}\right] \succeq 0,} \\
& \text { for all }(i, j):\left|\theta_{i j}\right| \leq 120^{\circ}, \quad i=1, \ldots, n-1 \text {, } \\
& j=2, \ldots, n, \\
& {\left[\begin{array}{cc}
\Pi^{\mathrm{T}} \tilde{\mathrm{C}}_{i} & \Pi^{\mathrm{T}} \tilde{\mathrm{T}}_{i j} \\
\Pi^{\mathrm{T}} \tilde{\mathrm{T}}_{i j} & -\Pi^{\mathrm{T}} \tilde{\mathrm{T}}_{j i}
\end{array}\right] \succeq 0,} \\
& {\left[\begin{array}{ll}
\Pi^{\mathrm{T}} \tilde{\mathrm{C}}_{j} & \Pi^{\mathrm{T}} \tilde{\mathrm{T}}_{j i} \\
\Pi^{\mathrm{T}} \tilde{\mathrm{T}}_{j i} & -\Pi^{\mathrm{T}} \tilde{\mathrm{T}}_{i j}
\end{array}\right] \succeq 0,} \\
& \text { for all }(i, j):\left|\theta_{i j}\right| \geq 120^{\circ}, \quad i=1, \ldots, n-1 \text {, } \\
& j=2, \ldots, n \text {. }
\end{aligned}
$$


Note that 177 without the LMI constraints reduces to the QUARC LP problem. A QUARCH may be extended to preserve the convex hull of the set of scene points (see Figure 1), by simply augmenting the SDP problem in (17) with the corresponding linear inequalities for the scene points.

\subsection{Camera self-calibration with QUARCH}

As a specialization of a QUARC, a QUARCH comes one step closer to an affine reconstruction. This forms the basis of our camera self-calibration algorithm, where we use a QUARCH plane as an initialization for local optimization of a suitable cost function to locate $\Pi_{\infty}$. We propose using a normalized version of the cost function in [20]:

$$
\mathcal{F}\left(\Pi_{\infty}\right)=\sum_{i=1}^{n-1} \sum_{j=i+1}^{n}\left(\frac{\mathcal{M}_{i j}}{\left(\Pi_{\infty}^{\mathrm{T}} \tilde{\mathrm{C}}_{i}\right)^{2}\left(\Pi_{\infty}^{\mathrm{T}} \tilde{\mathrm{C}}_{j}\right)^{2}}\right)^{2},
$$

where $\mathcal{M}_{i j}$ is the modulus constraint polynomial in 3 and the normalization eliminates the scale factors from the cost. We optimize for the first three coordinates of $\Pi_{\infty}$, fixing the fourth to 1 . The calibration parameters are obtained after linearly estimating the dual image of the absolute conic.

To compute a QUARCH plane, we assume that the relative orientation angle is under $120^{\circ}$ between consecutive views. This is a mild assumption in practice that is often satisfied in image sequences acquired for the purpose of feature matching and $3 \mathrm{D}$ reconstruction. We solve the following SDP problem to obtain a QUARCH plane $\Pi_{\mathrm{Q}}$ :

$$
\begin{array}{ll}
\max _{\Pi, \mathrm{z}} & \log \operatorname{det} \mathrm{Z} \\
\text { s.t. } & \mathrm{Z} \succeq 0, \\
\quad-1 \leq(\Pi)_{k} \leq 1, & k=1, \ldots, 4, \\
{\left[\begin{array}{cc}
\Pi^{\top} \tilde{\mathrm{C}}_{i} & \Pi^{\mathrm{T}} \tilde{\mathrm{T}}_{i j} \\
\Pi^{\mathrm{T}} \tilde{\mathrm{T}}_{i j} & 3 \Pi^{\mathrm{T}} \tilde{\mathrm{T}}_{j i}
\end{array}\right] \succeq \mathrm{Z}, \quad\left[\begin{array}{cc}
\Pi^{\mathrm{T}} \tilde{\mathrm{C}}_{j} & \Pi^{\mathrm{T}} \tilde{\mathrm{T}}_{j i} \\
\Pi^{\mathrm{T}} \tilde{\mathrm{T}}_{j i} & 3 \Pi^{\mathrm{T}} \tilde{\mathrm{T}}_{i j}
\end{array}\right] \succeq \mathrm{Z},} \\
& i=1, \ldots, n-1, j=i+1 .
\end{array}
$$

Problem (19) can be efficiently solved using an interiorpoint method. Maximizing $\log \operatorname{det} \mathrm{Z}$ prevents the terms $\Pi^{\mathrm{T}} \tilde{\mathrm{T}}_{i j}$ and $\Pi^{\mathrm{T}} \tilde{\mathrm{T}}_{j i}$ from being arbitrarily close to zero, which is the case for $\Pi_{\infty}$ as $\left|\theta_{i j}\right|$ approaches $120^{\circ}$. From our empirical tests, a QUARCH plane from this SDP converges more reliably to the sought $\Pi_{\infty}$ in our algorithm than one from (17). Note that the QUARC inequalities are enforced in (19) as all camera centers are covered by using all pairs of consecutive views. Given a projective reconstruction $\left\{\mathrm{P}_{i}, \mathrm{X}_{j}\right\}$, the steps of our self-calibration algorithm are:

(i) QUARCH: compute $\Pi_{Q}$ using (19) and upgrade to QUARCH as $\mathrm{P}_{i}^{\mathrm{Q}}=\mathrm{P}_{i} \mathrm{H}_{\mathrm{Q}}^{-1}, \mathrm{X}_{j}^{\mathrm{Q}}=\mathrm{H}_{\mathrm{Q}} \mathrm{X}_{j}$,

(ii) Affine: locate $\Pi_{\infty}$ by minimizing (18) with $\mathrm{H}_{Q}^{-\mathrm{T}} \Pi_{Q}$ as initialization, and upgrade to affine as $\mathrm{P}_{i}^{\mathrm{A}}=$ $\mathrm{P}_{i}^{\mathrm{Q}} \mathrm{H}_{\mathrm{A}}^{-1}, \mathrm{X}_{j}^{\mathrm{A}}=\mathrm{H}_{\mathrm{A}} \mathrm{X}_{j}^{\mathrm{Q}}$, (iii) Metric: compute calibration $\mathrm{K}$ as [12, Sect. 19.5.2] and upgrade to metric as $\mathrm{P}_{i}^{\mathrm{M}}=\mathrm{P}_{i}^{\mathrm{A}} \mathrm{H}_{\mathrm{M}}^{-1}, \mathrm{X}_{j}^{\mathrm{M}}=\mathrm{H}_{\mathrm{M}} \mathrm{X}_{j}^{\mathrm{A}}$, with

$$
\mathrm{H}_{\mathrm{Q}}=\left[\begin{array}{l}
\tilde{\mathrm{P}}_{1} \\
\Pi_{\mathrm{Q}}^{\mathrm{T}}
\end{array}\right], \mathrm{H}_{\mathrm{A}}=\left[\begin{array}{c}
\mathrm{I}_{3} 0_{3} \\
\Pi_{\infty}^{\top}
\end{array}\right], \mathrm{H}_{\mathrm{M}}=\left[\begin{array}{cc}
\mathrm{K}^{-1} & 0_{3} \\
0_{3}^{\top} & 1
\end{array}\right] .
$$

\section{Constrained Levenberg-Marquardt method}

An unconstrained local optimization method to locate $\Pi_{\infty}$ in step (ii) of our algorithm may converge to a nonQUARCH plane, i.e. a plane that does not satisfy the LMIs in (11) for consecutive views, and is therefore not the sought $\Pi_{\infty}$. To ensure that these LMIs are satisfied during the local optimization, we propose a constrained LM method for nonlinear optimization subject to LMI constraints. Our approach is based on [13], where the optimization problem is:

$$
\min _{d}\left\|F\left(x_{k}\right)+J_{k} d\right\|^{2}+\mu_{k}\|d\|^{2} \text { s.t. } x_{k}+d \in \mathcal{C},
$$

that allows to compute a step $d$ such that the iterate $x_{k+1}=$ $x_{k}+d$ is in the convex set $\mathcal{C}$, where $\mathcal{F}(x)=\|F(x)\|^{2}$ is the natural merit function corresponding to the mapping $F(x)$, $J_{k}$ is the Jacobian of $F\left(x_{k}\right)$, and $\mu_{k}$ is a positive parameter at iteration $k$. Note that the quadratic objective function in (20) is strictly convex. This constrained LM method was shown in [13] to be locally quadratically convergent under a local error bound condition.

In our case, $x_{0}$ is the QUARCH plane initialization from (19), $\mathcal{C}$ is the subset of QUARCH planes, containing planes that satisfy the LMIs in (11) for consecutive views, and $\mathcal{F}(x)$ is the cost function used to locate $\Pi_{\infty}$ in (18). To compute a step $d$ such that the iterating plane $x_{k}$ remains in the set $\mathcal{C}$, first observe that $\left\|F_{k}+J_{k} d\right\|^{2}+\mu_{k}\|d\|^{2}$ expands as $F_{k}^{\top} F_{k}+2 F_{k}^{\top} J_{k} d+d^{\top}\left(J_{k}^{\top} J_{k}+\mu_{k} \mathrm{I}\right) d$, where $F_{k}$ is short for $F\left(x_{k}\right)$. Hence, problem (20) is equivalent to:

$$
\begin{array}{ll}
\min _{d, \delta} & \delta \\
\text { s.t. } & x_{k}+d \in \mathcal{C}, \\
& \delta-F_{k}^{\top} F_{k}-2 F_{k}^{\top} J_{k} d-d^{\top}\left(J_{k}^{\top} J_{k}+\mu_{k} \mathrm{I}\right) d \geq 0 .
\end{array}
$$

The inequality in 21) is quadratic in $d$ and can be reformulated into an LMI by applying Lemma 2.1. The step $d$ can then be computed by solving the following SDP problem:

$$
\begin{array}{ll}
\min _{d, \delta} & \delta \\
\text { s.t. } & {\left[\begin{array}{cc}
J_{k}^{\top} J_{k}+\mu_{k} \mathrm{I}_{3} & \left(J_{k}^{\top} J_{k}+\mu_{k} \mathrm{I}_{3}\right) d \\
d^{\top}\left(J_{k}^{\top} J_{k}+\mu_{k} \mathrm{I}_{3}\right) & \delta-F_{k}^{\top} F_{k}-2 F_{k}^{\top} J_{k} d
\end{array}\right] \succeq 0,} \\
& {\left[\begin{array}{cc}
\left(x_{k}+d\right)^{\top} \tilde{\mathrm{C}}_{i} & \left(x_{k}+d\right)^{\top} \tilde{\mathrm{T}}_{i j} \\
\left(x_{k}+d\right)^{\top} \tilde{\mathrm{T}}_{i j} & 3\left(x_{k}+d\right)^{\top} \tilde{\mathrm{T}}_{j i}
\end{array}\right] \succeq 0} \\
& {\left[\begin{array}{cc}
\left(x_{k}+d\right)^{\top} \tilde{\mathrm{C}}_{j} & \left(x_{k}+d\right)^{\top} \tilde{\mathrm{T}}_{j i} \\
\left(x_{k}+d\right)^{\top} \tilde{\mathrm{T}}_{j i} & 3\left(x_{k}+d\right)^{\top} \tilde{\mathrm{T}}_{i j}
\end{array}\right] \succeq 0} \\
& i=1, \ldots, n-1, j=i+1 .
\end{array}
$$


Note that the term $J_{k}^{\top} J_{k}+\mu_{k} \mathrm{I}_{3}$ in the $1^{\text {st }} \mathrm{LMI}$ in 22] is positive definite by construction. As with the unconstrained optimization approach, we optimize for the first three coordinates of $\Pi_{\infty}$, fixing the fourth to 1 . Thus, we compute the first three coordinates of the step $d$, the fourth being 0 .

We use $\mu_{k}=\mu\left\|F\left(x_{k}\right)\right\|$, rather than the squared norm, following the choice of Yu [27]. Fan [6] showed that the same quadratic convergence rate is obtained with this choice. Our constrained LM method ensures that the iterating plane remains in the subset of QUARCH planes to which $\Pi_{\infty}$ belongs. Doing so prevents the iterating plane from crossing the camera centers, which could be fatal for the cost function in (18). As a local optimization method, it remains susceptible to converge to a local minimum of the cost function, albeit one that is also a QUARCH plane.

\section{Experimental results}

We tested our self-calibration algorithm on synthetic data and real images using both unconstrained and constrained optimization. Projective reconstructions for the synthetic data were obtained using the implementation of [18] in [22], followed by a projective bundle adjustment. For the real image sequences, they were obtained using P2SfM [15] with COLMAP [25] for feature matching. Data normalization was used throughout. We set $\mu=0.5$ and used the update $\mu_{k+1}=\min \left\{\mu_{k}, \mu_{k}\left\|F\left(x_{k+1}\right)\right\|\right\}$. Our algorithm is implemented in MATLAB R2017b with the convex optimization problems modeled using YALMIP [14] and solved using MOSEK [16]. All experiments were conducted on an Intel Core i7 3.10GHz 32GB RAM system.

\subsection{Synthetic data}

Each synthetic scene consisted of 500 points scattered randomly within the unit sphere and imaged by cameras placed at a distance of 2.75-3.45 units from the sphere center and facing towards it. The cameras were then perturbed by a small random translation. The rotation angle $\theta_{i j}$ between consecutive views was sampled randomly from the range $\left[20^{\circ}, 60^{\circ}\right]$ to satisfy the assumption $\left|\theta_{i j}\right| \leq 120^{\circ}$. All cameras had (in pixels) focal length $f_{x}=f_{y}=300$, zero skew i.e. $\gamma=0$, and an image-centered principal point i.e. $\left(u_{0}, v_{0}\right)=(128,128)$. Zero-mean Gaussian noise with standard deviation in the $[0,2]$ pixel range was added to pixel coordinates in increments of 0.5 pixels. The sequence length was varied from 4 to 16 views, and 100 trials were run for each sequence length and image noise level.

We conducted a series of reliability tests to examine the benefits of the QUARCH LMIs in self-calibration. We evaluated our results using the 3D RMS error between the ground truth and the recovered metric point clouds (both scaled to have a mean distance of 1 unit) aligned by a bestfit similarity transformation in the least squares sense. In this section, we show the distribution of 3D errors from the metric upgrade using box plots (following MATLAB's convention). To aid in visualization, we compressed samples beyond 0.02 3D error uniformly in a small region beyond this limit (shown by a dashed line), while preserving their relative order. We also scattered all points by a small random amount to distinguish between the overlapping ones.

QUARCH vs. QUARC: We compared a QUARCH plane initialization with a QUARC using an unconstrained minimization of (18), denoted as QUARCH-M and QUARC$\mathrm{M}$, respectively (Figure 2). Considering $0.02 \mathrm{3D}$ error as a threshold for a successful metric upgrade, for 4-5 views in Figure 2, the significant difference in the number of error points beyond this limit shows that a QUARCH plane converged to the true $\Pi_{\infty}$ more often than a QUARC and therefore retrieved a metric reconstruction more reliably. Consequently, QUARCH-M led to a considerably smaller median error, particularly for higher levels of pixel noise. As the sequence length increased to 6 views, both QUARCH-M and QUARC-M succeeded most of the time. We also show results for both planes using Nistér's cost function [17], denoted similarly as QUARCH-N and QUARC-N (Figure 3 . Here as well, a QUARCH plane led to a metric upgrade more reliably, though the difference is less pronounced. This is because Nistér's cost function is based on strong camera priors, such as zero skew, unit aspect ratio, and principal point at the image center, properties that are fully satisfied by our simulated cameras. Hence, both planes successfully converged to the true $\Pi_{\infty}$ most of the time.

QUARCH* vs. QUARCH: We compared the results from QUARCH-M with those from constrained optimization, denoted as QUARCH*M. In Figure 2, QUARCH*M successfully recovered the metric structure for several projective reconstructions that had otherwise failed with unconstrained optimization. Enforcing the QUARCH LMIs during optimization led the QUARCH plane to reliably converge to the true $\Pi_{\infty}$, while avoiding to succumb to a non-QUARCH local minimum of the modulus constraints. QUARCH*M, on average, required 5 iterations to converge and took $<1.2 \mathrm{~s}$ up to 16 views, of which $<0.2 \mathrm{~s}$ was for computing the QUARCH plane. The runtime scaled linearly in the number of views as do the LMI constraints in our SDP problems in (19) and 22). Beyond 6 views, QUARCH-M sufficed for a successful metric upgrade and can be used instead for a speedup. With Nistér's cost in Figure 3 , we observed that only one additional projective reconstruction, for 6 views, was successfully upgraded to metric using constrained optimization, denoted as QUARCH*N. These results show the benefits of enforcing the QUARCH LMIs during the local optimization, particularly for short sequences and when using the modulus constraints.

QUARCH* vs. GO-DAQ and GO-Stratified: We compared QUARCH*M and QUARCH*N with two globally optimal methods: GO-DAQ [4] and GO-Stratified [5]. 

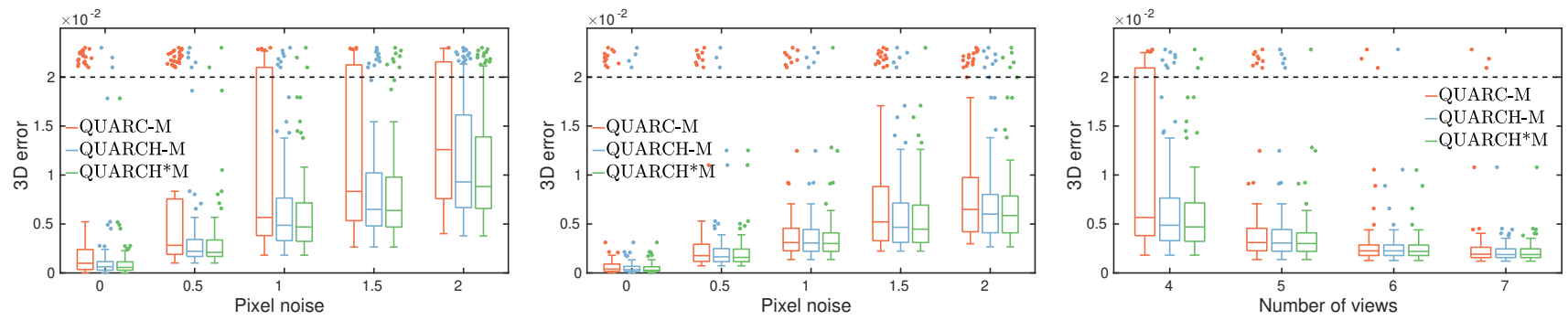

Figure 2: Comparison among QUARC-M, QUARCH-M, and QUARCH*M. Experiments using 4 views (left) and 5 views (middle) with varying noise levels, and using a varying number of views with 1 pixel of noise (right).
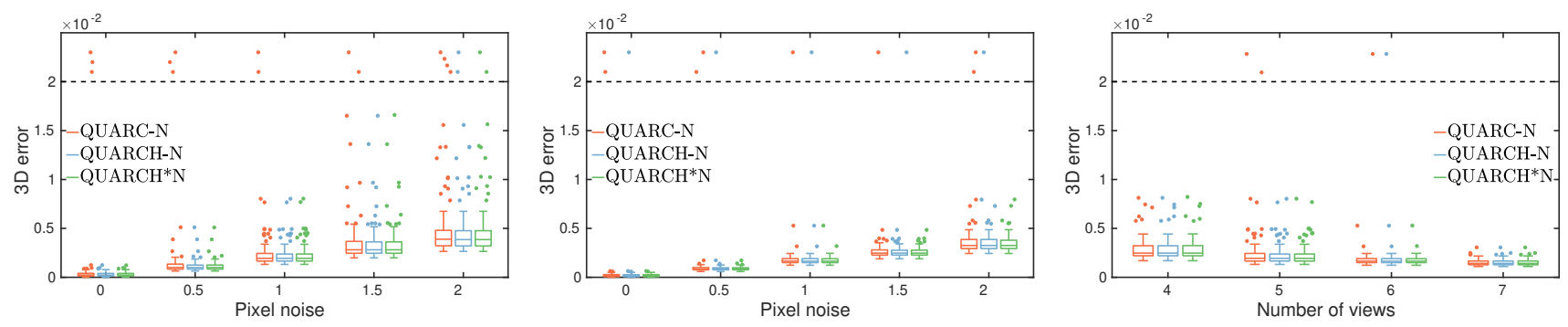

Figure 3: Comparison among QUARC-N, QUARCH-N, and QUARCH*N. Experiments using 5 views (left) and 6 views (middle) with varying noise levels, and using a varying number of views with 1 pixel of noise (right).

For GO-Stratified, we computed the calibration for both cheirality signs, and picked the resulting calibration closest to the ground truth (the authors' implementation was used). For GO-DAQ, we fixed the relaxation order to 2 , and used MOSEK as the solver. In the results shown in Figure 4. QUARCH*M consistently outperformed GOStratified, both in terms of the median 3D error and success rate. With 4-5 views, GO-Stratified failed frequently for noise levels above 1 pixel. This is likely because the modulus constraints admit multiple global solutions, and with short sequences there are fewer constraints to isolate the true $\Pi_{\infty}$. Also, this method relies on scene points to compute bounds for $\Pi_{\infty}$, which may prove to be unreliable in the presence of noise. With more views, the additional modulus constraints led to a more reliable calibration with GO-Stratified, but the median error was still larger than with QUARCH*M. The two methods with geometric cost functions (QUARCH*N and GO-DAQ) generally outperformed the other two, however, GO-DAQ suffered from a drastic increase in 3D error for high levels of pixel noise. The likely explanation for this result is that our simulated cameras approach a known "artificial" degenerate configuration for estimating the Dual Absolute Quadric (DAQ). This degenerate configuration occurs when all optical axes pass through a common point [7] and the rank of the DAQ is not enforced. Our cameras approach such a configuration in the presence of noise. GO-DAQ, because of scaling and numerical tractability issues (cost and constraints are unnor- malized), is then likely to fail as the rank-3 constraint on the DAQ is not earnestly enforced. As the sequence length increased, in Figure 4, all methods performed fairly reliably.

\subsection{Real images}

We present results on six real image sequences: fountain-P11, Herz-Jesu-P8, and Herz-Jesu-P25 from [26], Vercingetorix and Alcatraz water tower from [19], and Cherub [1]. The first three provide the ground truth calibration with focal lengths $f_{x}^{t}=2759.48, f_{y}^{t}=2764.16$, principal point $\left(u_{0}^{t}, v_{0}^{t}\right)=(1520.69,1006.81)$, and skew $\gamma^{t}=0$ pixels. For these three sequences, we computed the following errors for a quantitative evaluation: focal length error $\Delta f=\left|f_{x}^{t}-f_{x}\right|+\left|f_{y}^{t}-f_{y}\right|$, principal point error $\Delta u v=\left|u_{0}^{t}-u_{0}\right|+\left|v_{0}^{t}-v_{0}\right|$, and skew error $\Delta \gamma=$ $\left|\gamma^{t}-\gamma\right|$. For the remaining three sequences, we analyzed the recovered metric reconstructions qualitatively. Our experiments also served to verify the practical applicability of our assumption that $\left|\theta_{i j}\right| \leq 120^{\circ}$ for consecutive views.

Quantitative evaluation: From the calibration errors reported in Table 1. QUARC-M and GO-Stratified on the Herz-Jesu-P8 sequence, and QUARC-N on the Herz-Jesu$P 25$ sequence, led to an erroneous calibration. Upon inspection, their corresponding reconstructions failed to achieve a metric upgrade and remained projectively distorted. Note that QUARCH*M succeeded on the Herz-Jesu$P 8$ sequence, whereas the other two methods, also based on the modulus constraints, failed. This confirms our re- 

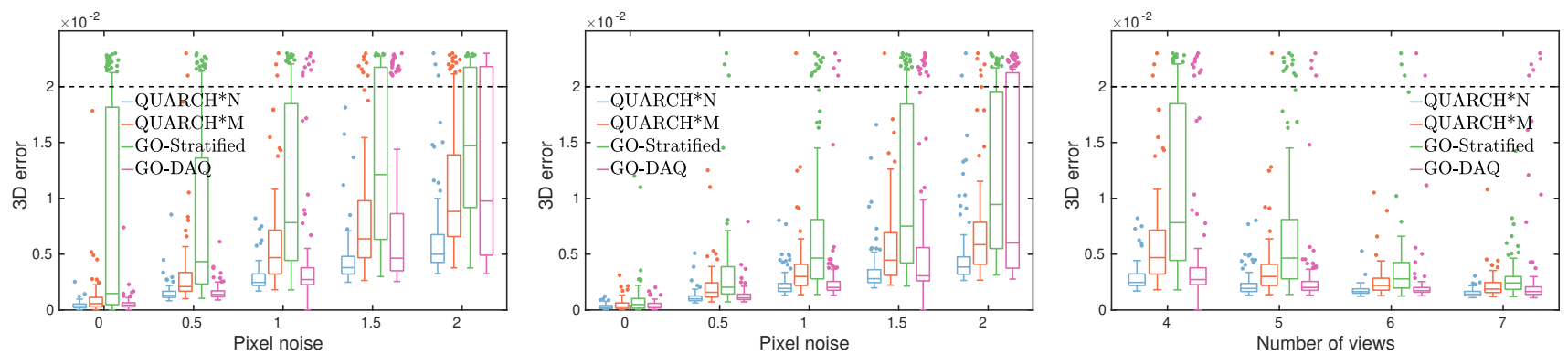

Figure 4: Comparison of QUARCH*N and QUARCH*M with GO-Stratified and GO-DAQ. Experiments using 4 views (left) and 5 views (middle) with varying noise levels, and using a varying number of views with 1 pixel of noise (right).
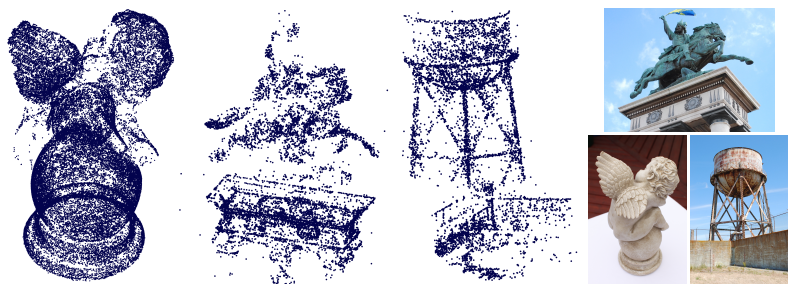

Figure 5: 3D reconstructions of (from left to right) Cherub, Vercingetorix, and Alcatraz water tower obtained with QUARCH*M. Sample images shown on the right.

sults with the synthetic data that the QUARCH LMIs aid in reliably locating the true $\Pi_{\infty}$ and that GO-Stratified often fails for short sequences. Except for these failures, all the methods otherwise led to a calibration close to ground truth and thereby to a successful metric upgrade. The error measurements are not completely indicative of the reconstruction quality, which, from our observations, is primarily influenced by the focal length and skew errors. From the timing results in Table 1 1 , the unconstrained local optimization methods are considerably faster than the others. The constrained optimization method is slower than the unconstrained one due to the more expensive SDP problem computation at each iteration. GO-DAQ took a similar amount of time as QUARCH*M and QUARCH*N, but GOStratified was significantly slower for all tested sequences.

Qualitative evaluation: We show the 3D reconstruction results obtained with QUARCH*M on three longer image sequences: Cherub, Vercingetorix, and Alcatraz water tower in Figure 5. These sequences have 65, 69, and $173 \mathrm{im}-$ ages, respectively, and their corresponding projective reconstructions contained 65, 63, and 66 cameras, respectively. The recovered metric structures closely resemble the captured scenes. Similar metric reconstructions were obtained using QUARCH*N. We observed that several points were poorly estimated in these reconstructions. These led to failures with GO-Stratified as it relies on all scene points. Our results also confirm the applicability of the assumption of

\begin{tabular}{llrrrr}
\hline Sequence & Method & \multicolumn{1}{c}{$\Delta f$} & \multicolumn{1}{c}{$\Delta u v$} & \multicolumn{1}{c}{$\Delta \gamma$} & Time (s) \\
\hline fountain-P11 & QUARCH*M & 1.91 & 4.01 & 0.99 & 2.71 \\
& QUARC-M & 2.44 & 4.30 & 0.99 & 0.09 \\
& QUARCH*N & 42.92 & 28.29 & 0.71 & 1.47 \\
& QUARC-N & 43.73 & 28.61 & 0.69 & 0.10 \\
& GO-DAQ & 76.15 & 31.92 & 0.10 & 1.27 \\
& GO-Stratified & 12.64 & 9.75 & 1.17 & 449.47 \\
Herz-Jesu-P8 & QUARCH*M & 53.49 & 78.68 & 1.56 & 1.32 \\
& QUARC-M & 4114.66 & 101.16 & 586.29 & 0.07 \\
& QUARCH*N & 83.61 & 33.93 & 1.24 & 1.90 \\
& QUARC-N & 76.81 & 34.22 & 1.21 & 0.09 \\
& GO-DAQ & 66.10 & 33.84 & 0.27 & 1.85 \\
& GO-Stratified & 2552.62 & 1006.05 & 132.85 & 154.74 \\
Herz-Jesu-P25 & QUARCH*M & 34.89 & 23.71 & 2.52 & 2.04 \\
& QUARC-M & 34.75 & 23.70 & 2.55 & 0.45 \\
& QUARCH*N & 59.04 & 31.44 & 1.40 & 2.08 \\
& QUARC-N & 2812.61 & 185.73 & 21.86 & 0.46 \\
& GO-DAQ & 4.40 & 33.90 & 0.60 & 1.60 \\
& GO-Stratified & 52.94 & 32.16 & 1.69 & 893.76 \\
\hline
\end{tabular}

Table 1: Self-calibration results on sequences from [26].

$\left|\theta_{i j}\right| \leq 120^{\circ}$ for consecutive views. This assumption might as well be extended to every other view in these sequences.

\section{Conclusion}

We presented a new quasi-affine reconstruction stratum, QUARCH, as a specialization of QUARC. We showed that $\Pi_{\infty}$ satisfies either of two sets of LMIs for a camera pair depending on the relative orientation angle being under or over $120^{\circ}$. We also proposed a constrained LM method to enforce the QUARCH LMIs during the local optimization to locate $\Pi_{\infty}$. Our experiments showed the benefits of the QUARCH LMIs in reliably locating $\Pi_{\infty}$ to obtain a metric reconstruction from a projective one. Our constrained LM method could also be useful in other computer vision problems for nonlinear refinement subject to LMI constraints.

Acknowledgements: This research was partially supported by the ANR SUMUM project, grant ANR-17-CE38-0004. 


\section{References}

[1] 3Dflow SRL. 3DF Zephyr Reconstruction Showcase. https://www.3dflow.net/ 3df-zephyr-reconstruction-showcase/

[2] Max K. Agoston. Computer Graphics and Geometric Modelling: Implementation \& Algorithms. Springer, 2005.

[3] Stephen Boyd and Lieven Vandenberghe. Convex Optimization. Cambridge University Press, 2004.

[4] Manmohan Chandraker, Sameer Agarwal, Fredrik Kahl, David Nistér, and David Kriegman. Autocalibration via Rank-Constrained Estimation of the Absolute Quadric. In Proceedings of the IEEE Conference on Computer Vision and Pattern Recognition, pages 1-8, 2007.

[5] Manmohan Chandraker, Sameer Agarwal, David Kriegman, and Serge Belongie. Globally Optimal Algorithms for Stratified Autocalibration. International Journal of Computer Vision, 90(2):236-254, 2010.

[6] Jinyan Fan. On the Levenberg-Marquardt methods for convex constrained nonlinear equations. Journal of Industrial and Management Optimization, 9(1):227-241, 2013.

[7] Pierre Gurdjos, Adrien Bartoli, and Peter F. Sturm. Is dual linear self-calibration artificially ambiguous? In Proceedings of the IEEE International Conference on Computer Vision, pages 88-95, 2009.

[8] Adlane Habed, Kassem Al Ismaeil, and David Fofi. A New Set of Quartic Trivariate Polynomial Equations for Stratified Camera Self-calibration under Zero-Skew and Constant Parameters Assumptions. In Proceedings of the European Conference on Computer Vision, pages 710-723. Springer Berlin Heidelberg, 2012.

[9] Richard Hartley. Euclidean reconstruction from uncalibrated views. In Proceedings of the Joint European-US Workshop on Applications of Invariance in Computer Vision, pages 235-256, 1994.

[10] Richard Hartley. Chirality. International Journal of Computer Vision, 26(1):41-61, 1998.

[11] Richard Hartley, Eric Hayman, Lourdes de Agapito, and Ian Reid. Camera calibration and the search for infinity. In Proceedings of the IEEE International Conference on Computer Vision, pages 510-517, 1999.

[12] Richard Hartley and Andrew Zisserman. Multiple View Geometry in Computer Vision. Cambridge University Press, 2nd edition, 2004.

[13] Christian Kanzow, Nobuo Yamashita, and Masao Fukushima. Levenberg-Marquardt methods with strong local convergence properties for solving nonlinear equations with convex constraints. Journal of Computational and Applied Mathematics, 172(2):375-397, 2004.

[14] Johan Löfberg. YALMIP: A toolbox for modeling and optimization in MATLAB. In Proceedings of the IEEE International Conference on Robotics and Automation, pages 284289, 2004.

[15] Ludovic Magerand and Alessio Del Bue. Practical Projective Structure from Motion (P2SfM). In Proceedings of the IEEE International Conference on Computer Vision, pages 39-47, 2017.
[16] MOSEK ApS. The MOSEK optimization toolbox for MATLAB manual. Version 8.1. http://docs.mosek.com/ 8.1/toolbox/index.html 2017.

[17] David Nistér. Untwisting a Projective Reconstruction. International Journal of Computer Vision, 60(2):1-33, 2004.

[18] John Oliensis and Richard Hartley. Iterative Extensions of the Sturm/Triggs Algorithm: Convergence and Nonconvergence. IEEE Transactions on Pattern Analysis and Machine Intelligence, 29(12):2217-2233, 2007.

[19] Carl Olsson and Olof Enqvist. Stable Structure from Motion for Unordered Image Collections. In Proceedings of the Scandinavian Conference on Image Analysis, pages 524535, 2011.

[20] Marc Pollefeys and Luc Van Gool. Stratified self-calibration with the modulus constraint. IEEE Transactions on Pattern Analysis and Machine Intelligence, 21(8):707-724, 1999.

[21] Helmut Pottmann and Johannes Wallner. Computational Line Geometry. Springer, 2010.

[22] Vincent Rabaud. Vincent's Structure from Motion Toolbox. http://github.com/vrabaud/sfm_toolbox

[23] José Ignacio Ronda, Antonio Valdés, and Fernando Jaureguizar. Camera Autocalibration and Horopter Curves. International Journal of Computer Vision, 57(3):219-232, 2004.

[24] Frederik Schaffalitzky. Direct Solution of Modulus Constraints. In Proceedings of the Indian Conference on Computer Vision, Graphics and Image Processing, pages 314321, 2000.

[25] Johannes Schönberger and Jan-Michael Frahm. Structurefrom-Motion Revisited. In Proceedings of the IEEE Conference on Computer Vision and Pattern Recognition, pages 4104-4113, 2016.

[26] Christoph Strecha, Wolfgang Von Hansen, Luc Van Gool, Pascal Fua, and Ulrich Thoennessen. On Benchmarking Camera Calibration and Multi-View Stereo for High Resolution Imagery. In Proceedings of the IEEE Conference on Computer Vision and Pattern Recognition, pages 1-8, 2008.

[27] Zhensheng Yu. On the global convergence of a LevenbergMarquardt method for constrained nonlinear equations. Journal of Applied Mathematics and Computing, 16(1):183194, 2004. 